\title{
Comunicación
}

\section{Pododermatitis ulcerativa severa infectada con Staphylococcus aureus y Proteus spp en un cuy (Cavia porcellus): reporte de caso}

\author{
Severe ulcerative pododermatitis infected with Staphylococcus aureus and Proteus \\ spp in a guinea pig (Cavia porcellus): a case report
}

Renzo Venturo B. ${ }^{1}$; Siever Morales-Cauti ${ }^{1,2}$

\section{Resumen}

Se describe un caso de pododermatitis ulcerativa severa infectada con Staphylococcus aureus y Proteus spp en un cuy criado en jaula metálica. Un cuy hembra de 850 g y 9 meses de edad es llevado a consulta por presentar molestias al caminar. Se diagnosticó pododermatitis ulcerativa severa (grado IV o V) en extremidades posteriores basándose en signos clínicos, citología y radiografías. Se retiró al animal de la jaula y fue colocado en superficie plana. Se le trató con un preparado a base de $4 \mathrm{~g}$ de azúcar y $0.5 \mathrm{ml}$ de sangre de grado (Croton lechleri), aplicándose en la lesión una vez al día y con posterior vendaje del área afectada; además se utilizó prednisolona oral a dosis de $0.8 \mathrm{mg} / \mathrm{kg}$ de p.v. cada 24 horas en jarabe por 14 días, y gentamicina subcutánea a dosis de $6 \mathrm{mg} / \mathrm{kg}$ p.v. cada 24 horas por un mínimo de 21 días. Se hicieron evaluaciones clínicas y de citología semanales. La paciente mejoró gradualmente, reduciendo las lesiones, el número de bacterias y de células inflamatorias. Las úlceras de la pata izquierda cerraron en el día 20 y de la pata derecha en el día 26 del tratamiento.

Palabras clave: cobayo, Staphylococcus aureus, pododermatitis ulcerativa, dermatitis, hipertrofia

\footnotetext{
${ }^{1}$ Carrera de Medicina Veterinaria y Zootecnia, Universidad Cientifica del Sur, Lima, Perú

${ }^{2}$ Email: sieverm@hotmail.com
}

Recibido: 15 de septiembre de 2020

Aceptado para publicación: 10 de julio de 2021

Publicado: 24 de agosto de 2021

CLos autores. Este artículo es publicado por la Rev Inv Vet Perú de la Facultad de Medicina Veterinaria, Universidad Nacional Mayor de San Marcos. Este es un artículo de acceso abierto, distribuido bajo los términos de la licencia Creative Commons Atribución 4.0 Internacional (CC BY 4.0) [https:// creativecommons.org/licenses/by/4.0/deed.es] que permite el uso, distribución y reproducción en cualquier medio, siempre que la obra original sea debidamente citada de su fuente original 
A case of severe ulcerative pododermatitis infected with Staphylococcus aureus and Proteus spp in a guinea pig raised in a metal cage is described. A9-month-old female guinea pig weighing $850 \mathrm{~g}$ is taken to consultation for presenting discomfort when walking. Severe ulcerative pododermatitis (grade IV or V) in the hind limbs was diagnosed based on clinical signs, cytology, and radiographs. The animal was removed from the cage and placed on a flat surface. She was treated with a preparation based on $4 \mathrm{~g}$ of sugar and 0.5 $\mathrm{ml}$ of «dragon's blood» (Croton lechleri), applying it to the lesion once a day and subsequently bandaging the affected area; in addition, oral prednisolone was used at a dose of $0.8 \mathrm{mg} / \mathrm{kg}$ every 24 hours in syrup for 14 days, and subcutaneous gentamicin at a dose of $6 \mathrm{mg} / \mathrm{kg}$ every 24 hours for a minimum of 21 days. Weekly clinical and cytology evaluations were made. The patient gradually improved, reducing the lesions, and the number of bacteria and inflammatory cells. The ulcers on the left leg closed on day 20 and on the right leg on day 26 of treatment.

Key words: guinea pig, Staphylococcus aureus, ulcerative pododermatitis, dermatitis, hypertrophy

\section{INTRODUCCIÓN}

El cobayo (Cavia porcellus) también conocido como cuy, es un roedor histricomorfo que pertenece al orden Rodentia, familia Caviidae y género Cavia. Esta especie, usada como fuente de alimento y parte de ceremonias religiosas, se encuentra distribuida globalmente como mascota o animal de experimentación, siendo considerada una fuente de proteína de origen animal en países de la comunidad andina (Morales, 2013; Fawcett, 2015; Minarikova et al., 2015; Pignon y Mayer, 2020).

Este animal es considerado como especie exótica porque su frecuencia no es muy común en la práctica clínica; además, especie no requiere vacunas a diferencia de perros, gatos, conejos y hurones (Fawcett, 2015). Entre las enfermedades dermatoló-gicas descritas en cobayos o cuyes se encuentra la pododermatitis, conocida también como «bumblefoot» o «sore hocks», dermatitis crónica en las superficies plantares o podales, que en casos severos su pronóstico es de reservado a malo, debido al poco éxito esperado del tratamiento (Patterson, 2006; Brown y Donnelly, 2008; Blair, 2013; Fawcett, 2015; Pignon y Mayer, 2020).

Se presenta un caso de pododermatitis crónica ulcerativa severa infectada con Staphylococcus aureus y Proteus spp en un cuy criado en jaula metálica, tratado a base de sangre de grado, azúcar, prednisolona y gentamicina.

\section{Descripción del Caso}

Un cuy hembra de $850 \mathrm{~g}, 9$ meses de edad, es llevado a consulta por presentar molestias al caminar y por lesiones dermatológicas en las superficies plantares de los miembros posteriores. Según el propietario, el animal es alimentado a base de forraje verde únicamente, le realizó un cambio de hábitat a jaula desde hace tres semanas, las lesiones plantares tienen una evolución de dos semanas, y el apetito es normal. El estado conductual del animal es en condición de alerta. Al examen físico no se encontraron alteraciones respecto a frecuencia cardiaca y respiratoria o temperatura corporal. 


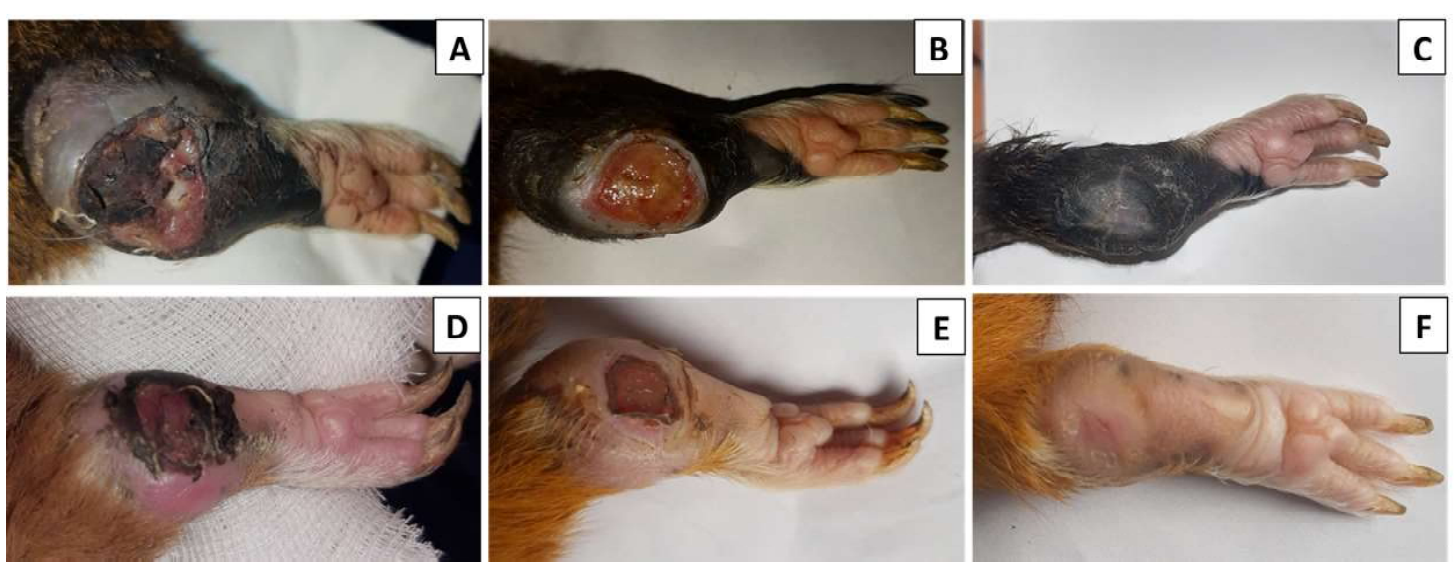

Figura 1. Evolución de las lesiones podales en un cuy. A) Superficie plantar izquierda día 1; B) día 14; C) Día 26; y E) superficie plantar derecha día 1; F) día 4 y G= día 26 después del tratamiento
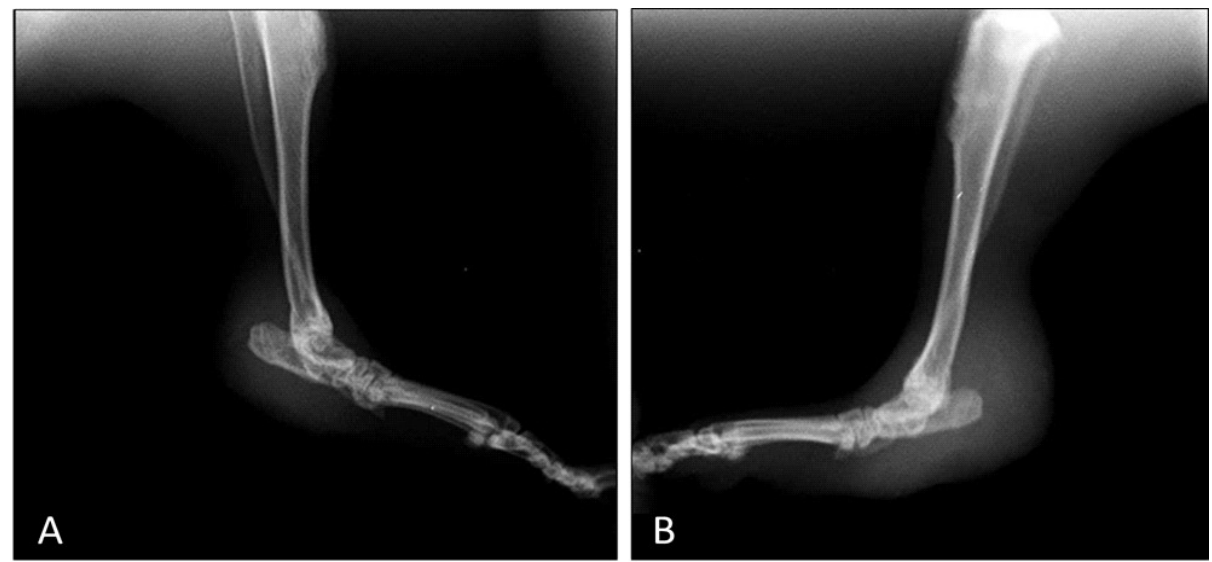

Figura 2. Radiografias de miembros posteriores de cuy con dermatitis plantar. Vista medio lateral. Aumento de densidad alrededor de la articulación tarsal en la pata derecha (A) e izquierda (B)

En la superficie plantar de la pata trasera derecha se evidenciaron lesiones erosivas ulcerativas, sangrantes, tejido necrótico, incremento de tejido alrededor de la lesión y sólido a la palpación (Figura 1). En la superficie plantar de la pata trasera izquierda se apreciaron lesiones similares, pero de menor extensión y profundidad. Los ganglios poplíteos de ambas extremidades se encontraban hiperplásicos. De otra parte, no se encontraron lesiones en la superficie palmar de las patas anteriores.
El diagnóstico se inició con una citología por hisopado, cultivo bacteriano, antibiograma y radiografía. En la citología se obtuvieron bacterias con morfología de cocos $3+$ y bacilos $3+$ extra e intracelulares con heterófilos $3+$, según la escala semicuantitativa descrita por Budach y Mueller (2012). Al confirmarse la infección se realizó un cultivo y antibiograma con resultado de Staphylococcus aureus y Proteus spp, el primero sensible a todos los antibióticos evaluados 


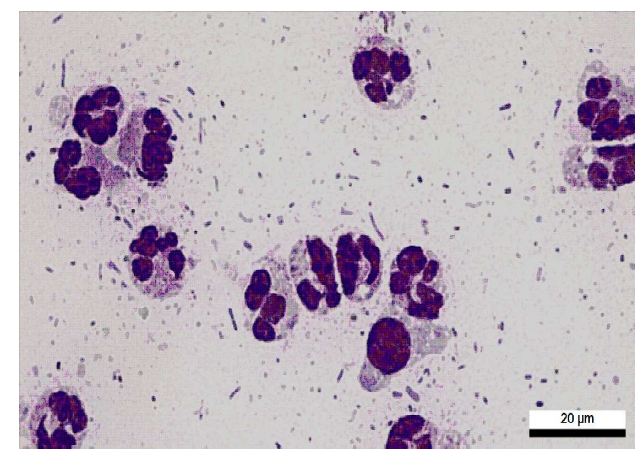

Figura 3. Citología de lesión en cuy con dermatitis plantar, compatible con proceso inflamatorio supurativo. Se observan heterofilos, bacterias con morfología cocoide y bacilar

mientras que el segundo fue resistente a Cefalexina, Cloranfenicol, Norfloxacina, Enrofloxacina, Ciprofloxacina, y sensible a Gentamicina, Amikacina e Imipenem.

En las radiografías, la vista medio-lateral de ambas extremidades posteriores mostraron estructuras óseas conservadas; sin embargo, se evidenció aumento de densidad en articulaciones tarsales sugerentes a sinovitis, siendo más notorio en la pata trasera derecha (Figura 2). No se apreció osteomielitis.

Basado en la escala propuesta por White et al. (2016), se diagnosticó pododermatitis ulcerativa severa (grado 4 o 5) en ambas extremidades. Como tratamiento se recomendó retirar al animal de la jaula y colocarlo a superficie plana manteniendo una higiene adecuada. Se utilizó un preparado mezclando $4 \mathrm{~g}$ de azúcar y $0.5 \mathrm{ml}$ de sangre de grado (Croton lechleri), donde $1 \mathrm{ml}$ de sangre de grado equivale a $1 \mathrm{ml}$ de látex de $C$. lechleri, aplicándose en la lesión una vez al día y posterior vendaje del área afectada. Además, se aplicó prednisolona oral a dosis de $0.8 \mathrm{mg} / \mathrm{kg}$ cada 24 horas en jarabe por 14 días y gentamicina subcutáneo a dosis de $6 \mathrm{mg} / \mathrm{kg}$ cada 24 horas por un mínimo de 21 días.
Se programaron controles semanales, evaluando las lesiones clínica y citológicamente (Figura 3). El paciente mejoró gradualmente, reduciendo el número de bacterias, células inflamatorias, al igual que las lesiones y su extensión. Se obtuvo el cierre completo de la úlcera el día 20 en la pata izquierda y el día 26 en la pata derecha.

\section{Discusión}

La pododermatitis ulcerativa suele ser de presentación esporádica y representa un problema para los animales criados en jaulas metálicas. Minarikova et al. (2015) reportó una frecuencia de 3\% (30/1000) y White et al. (2016) indicaron $43.7 \%$ (128/293) de casos de pododermatitis en una casuística de 25 años, aunque solo ocho casos fueron considerados como severos (grado IV o V). La patología es generalmente de origen bacteriano, donde influyen factores predisponentes como animales obesos mantenidos en condiciones sanitarias no óptimas, jaulas metálicas o abrasivas, astillas de madera, suciedad, deficiencia de vitamina $\mathrm{C}$ (Suckow et al., 2012; Blair, 2013; Pignon y Mayer, 2020). En el presente caso se debió probablemente a las características deficientes de material metálico en la jaula.

Los signos clínicos asociados a pododermatitis en cuyes son cojera, comportamiento sedentario, vocalización, pudiendo estar afectadas una o varias patas (Patterson, 2006; Clemons y Seeman, 2011; Suckow et al., 2012). Para Hoppman y Barron (2007), las técnicas recomendadas para el diagnóstico son la citología del exudado, la cual demuestra una inflamación piogranulomatosa con mayor presencia de cocos bacterianos, cultivo bacteriológico y radiografías, lo cual fue empleado con éxito en el presente caso. En el cultivo bacteriano se aislaron Staphylococcus aureus y Proteus spp, siendo $S$. aureus la bacteria más frecuentemente aislada en este tipo de cuadros (Rigby, 1976; Brown y Donnelly, 2008). No se encontraron 
reportes referentes a resistencia antibacteriana ante estos microorganismos; sin embargo, Proteus spp fue resistente a los antibióticos más usados para esta enfermedad como son la enrofloxacina $y$ ciprofloxacina (Brown y Donnelly, 2008).

La radiografía permitió tomar la decisión del tratamiento a realizar y la categorización de la lesión (Figura 2). No hay reportes sobre el uso de prednisolona para pododermatitis en cobayos, pero se manejó a dosis de $0.8 \mathrm{mg} / \mathrm{kg}$ de peso cada 24 horas por 14 días por vía oral, para reducir la inflamación. Con base a la sensibilidad antibiótica determinada, la duración de la terapéutica antibiótica sistémica puede establecerse por tres semanas (Hillier et al., 2014).

Este es el primer reporte del uso de combinación de azúcar con sangre de grado para la cicatrización en una pododermatitis ulcerativa severa en cobayos. El azúcar tiene propiedades higroscópicas con alta osmolaridad, que permite eliminar las bacterias mientras promueve el tejido de granulación, además mejora la migración de macrófagos, reducción de edema y epitelización; su uso se recomienda en forma de pasta por ser más efectiva y fácil aplicación (Pavletic, 2018). De otra parte, el látex de $C$. lechleri (sangre de grado) tiene propiedades inmunomoduladoras para heridas, capacidad para formar una película que proteja contra la invasión microbiana de heridas, actividad captadora de radicales libres brindadas por las procianidinas, efecto antiinflamatorio, antibacteriano y acción de los polifenoles (Jones, 2003; Risco et al., 2003).

\section{Literatura Citada}

1. Blair J. 2013. Boomblefoot: a comparison of clinical presentation and treatment of pododermatitis in rabbits, rodents, and birds. Vet Clin Exot Anim 16: 715-735. doi: 10.1016/j.cvex.2013.-05.002
2. Brown C, Donelly T. 2008. Treatment of pododermatitis in the guinea pig. Lab Animal 37: 156-157. doi: 10.1038/ laban0408-156

3. Budach S, Mueller RS.2012. Reproducibility of a semiquantitative method to assess. Vet Dermatol 23: 426-e80. doi: 10.1111/j.1365-3164.2012.-01075.x

4. Clemons DJ, Seeman JL. 2011. The laboratory guinea pig. $2^{\text {nd }} \mathrm{ed}$. USA: CRC Press. 176 p.

5. Fawcett A. 2015. Management of husbandry-related problems in guinea pigs. In Practice 33: 163-171. doi: 10.1136/inp.d1812

6. Hillier A, Lloyd DH, Scott J, Blondeau $J M$, Boothe D, Breitschwerdt E, Guardabassi L, et al. 2014. Guidelines for the diagnosis and antimicrobial therapy of canine superficial bacterial folliculitis (antimicrobial guidelines working group of the international society for companion animal infectious diseases). Vet Dermatol 25: 163-e43. doi: $10.1111 /$ vde. 12118

7. Hoppman E, Barron HW. 2007. Rodent dermatology. J Exot Pet Med 16: 238.255. doi: $10.1053 /$ j.jepm.2007.10.003

8. Jones K. 2003. Review of sangre de drago (Croton lechleri) - a South American tree sap in the treatment of diarrhea, inflammation, insect bites, viral infections, and wounds: traditional uses to clinical research. J Altern Complem Med 9: 877-896. doi: 10.1089/107555303771952235

9. Minarikova A, Hauptman $K, E$. Jeklova E, Z. Knotek Z, V. Jekl V. 2015. Diseases in pet guinea pigs: a retrospective study in 1000 animals. Vet Rec 177: 200. doi: 10.1136/vr.103053

10. Morales S. 2013. La sanidad en sistemas de crianza comercial de cuyes. En: XXXV Reunión Científica Anual de la Asociación Peruana de Producción Animal. Puno, Perú.

11. Patterson S. 2006. Skin diseases of exotic pets. Oxford: Blackwell. 247 p. 
12. Pavletic MM. 2018. Atlas of small animal wound management and reconstructive surgery. $4^{\text {th }}$ ed. USA: John Wiley. 78 p.

13. Pignon C, Mayer J. 2020. Guinea pigs. In: Quesenberry KF, Orcutt CJ, Mans C, Carpenter JW (eds). Ferrets, rabbits, and rodents: clinical medicine and surgery. $4^{\text {th }}$ ed. St. Louis, Missouri: Elsevier. p 271-297.

14. Rigby C. 1976. Natural infections in guinea pig. Lab Anim 10: 119-142. doi: 10.1258/002367776781071503

15. Risco E, Ghia F, Vila R, Iglesias J, Alvarez E, Cañigueral S. 2003. Immunomodu-latory activity and chemical characterisation of sangre de Drago
(Dragon's blood) from Croton lechleri. Planta Med 59: 785-794. doi: 10.1055/s2003-43208

16. Suckow MA, Stevens KA, Wilson RP. 2012. The laboratory rabbit, guinea pig, hamster, and other rodents. London: Elsevier. 699 p.

17. White SD, Sanchez-Migallon D, PaulMurphy J, Hawkins MG. 2016. Skin diseases in companion guinea pigs ( $\mathrm{Ca}$ via porcellus): a retrospective study of 293 cases seen at the Veterinary Medical Teaching Hospital, University of California at Davis (1990-2015). Vet Dermatol 27: 395-e100. doi: 10.1111/ vde. 12348 